MATEC Web of Conferences 8, 03004 (2013)

DOI: $10.1051 /$ matecconf $/ 20130803004$

(c) Owned by the authors, published by EDP Sciences, 2013

\title{
Avant-Garde Ultrafast Laser Writing
}

\author{
P. G. Kazansky, M. Beresna and M. Gecevičius \\ Optoelectronics Research Centre, University of Southampton, SO171BJ Southampton, UK \\ pgk@orc.soton.ac.uk
}

\begin{abstract}
Ultrafast laser processing of transparent materials reveals new phenomena. Reviewed, are recent demonstrations of 5D optical memory, vortex polarization and Airy beam converters employing self-assembled nanostructuring, ultrafast laser calligraphy and polarization writing control using pulses with tilted front.
\end{abstract}

Material processing with ultrafast lasers has attracted considerable interest due to new science and a wide range of applications from laser surgery, integrated optics and optofluidics to optical data storage, 3D micro- and nanostructuring [1-4]. A decade ago it has been discovered that under certain irradiation conditions ordered subwavelength structures with features smaller than $20 \mathrm{~nm}$ can be formed in the volume of silica glass [5,6]. The effect of nanograting formation has attracted considerable interest with proposals of applications ranging from nanofluidics [7] to polarization control devices [8]. The mechanism of nanograting formation still remains a puzzle [9].

More recently, the self-assembled sub-wavelength nanostructuring have been proposed for fabrication of radial, azimuthal and vortex polarization converters (Fig. 1) and rewritable polarization multiplexed optical memory $[9,10]$, where the information encoding is realized by means of two birefringence parameters, i.e. the slow axis orientation $\left(4^{\text {th }}\right.$ dimension) and retardance $\left(5^{\text {th }}\right.$ dimension), in addition to three spatial coordinates (Fig. 2$)$. The

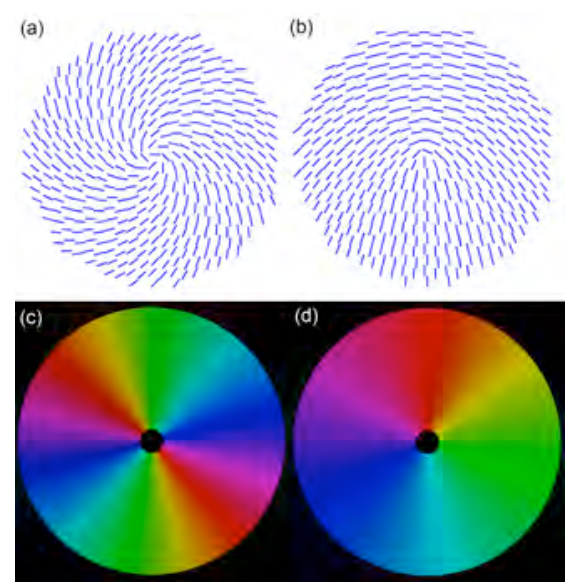

Fig. 1 Schematic drawings of nanogratings distribution in quarter- (a) and half-wave (b) polarization converters. Femtosecond laser written radial polarization converters for circular (c) and linear (d) incident polarizations. The pseudo color indicates direction of the slow axis.

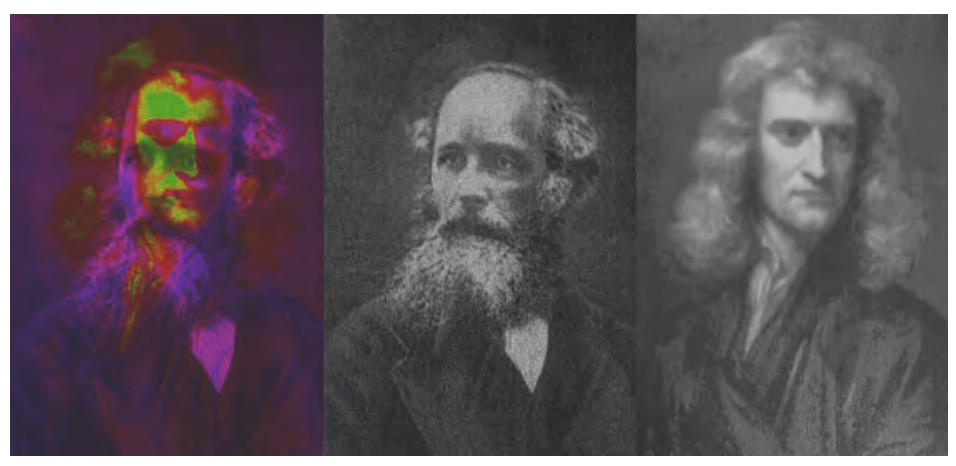

Fig. 2 Ultrafast laser optical recording via self-assembled nanograting induced birefringence in fused silica. Maxwell and Newton are recorded in one image (left, in pseudo colours), however, they can be easily decoupled as Maxwell is recorded in strength of retardance (centre) and Newton in azimuth of the slow axis (right). Size is $1.5 \times 2 \mathrm{~mm}$.

phase of the optical beam around a point, where the intensity is zero, e.g. in vortex beam, is undefined. This point is called phase singular point and the research field is named singular optics. We review recent progress in application of femtosecond laser nanostructuring of fused silica, in particular for direct printing of singular optics elements. The tight control of nanostructures' properties through writing parameters is demonstrated implementing elements with unique optical properties, in particular Southampton-Super-Structured-waveplates (S-waveplates [11]) for uniform to radial and azimuthal polarization conversion, which can be widely used in material processing, microscopy, fiber optics, optical trapping and manipulation.

More recently, we have demonstrated new technique to generate accelerating Airy beam with femtosecond laser imprinted space variant birefringence produced by self-assembled nanostructures in fused silica (Fig.3). The technique enabled dual Airy beam generation. 

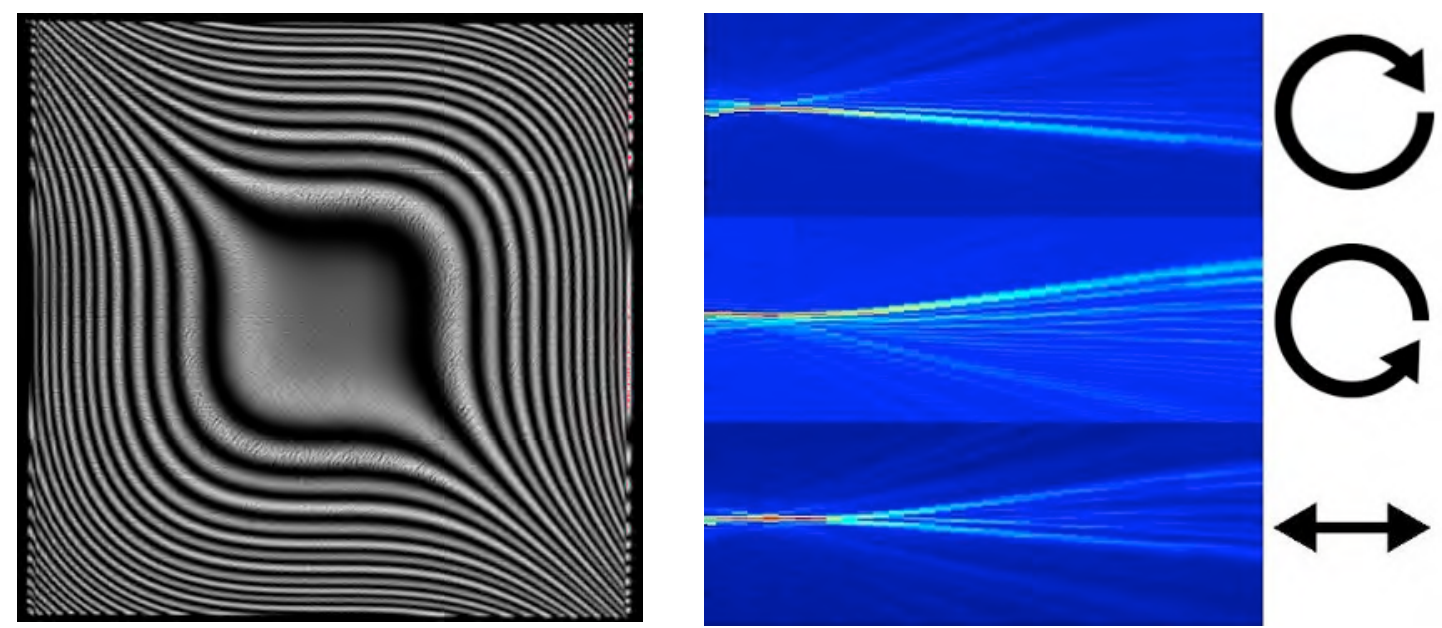

Fig. 3 Microscopic image of structure between cross-polarizers (left) Structure size is $2 \times 2 \mathrm{~mm}$. Propagation of 1D Airy beam (right). Arrow indicates the polarization of incident Gaussian beam.

A remarkable effect has also been discovered, referred to as quill or calligraphic laser writing, which reveals strong dependence of the material modification, in particular the self-assembled sub-wavelength structures in glass, on orientation of the writing direction relative to direction of the pulse front tilt [12-13]. Moreover, evidence of the first order phase transition associated with self-assembled nanostructures formation was revealed and supercooled state of laser damage in silica glass was observed using pulses with tilted intensity front. More recently it has been demonstrated that the tip of an ultrafast laser quill has a property that is very different from an ordinary quill [14]. Specifically, the modification of glass can be controlled even in stationary conditions by the mutual orientation of light polarization azimuth and the pulse front tilt. Figuratively, the polarization can be used as a sharpening blade for the ultrafast light quill. The demonstrations of self-assembled nano-structuring and employing mutual orientations of beam movement or the light polarization plane and pulse front tilt to control interaction of matter with ultrashort light pulses, open new opportunities in material processing.

\section{References}

[1] S.S. Mao, F. Quere, S. Guizard, X. Mao, R. E. Russo, G. Petite, and P. Martin, "Dynamics of femtosecond laser interaction with dielectrics", Appl. Phys. A 79, 1695 (2004).

[2] W. Yang, P. G. Kazansky, and Yu. P. Svirko, "Non-reciprocal ultrafast laser writing," Nature Photonics 2, 99 (2008).

[3] B. Poumellec, M. Lancry, J. Poulin, and S. Ani-Joseph, "Non-reciprocal writing and chirality in femtosecond laser irradiated silica," Opt. Express 16, 18354 (2008).

[4] Y. Bellouard, A. Said, M. Dugan, and P. Bado, "Fabrication of high-aspect ratio, micro-fluidic channels and tunnels using femtosecond laser pulses and chemical etching," Opt. Express 12, 2120 (2004).

[5] Y. Shimotsuma, P. G. Kazansky, J. Qiu, and K. Hirao, "Self-organized nanogratings in glass irradiated by ultrashort light pulses," Phys. Rev. Lett. 91, 247705 (2003).

[6] J. Canning, M. Lancry, K. Cook, A. Weickman, F. Brisset, and B. Poumellec, "Anatomy of a femtosecond laser processed silica waveguide", Optical Materials Express, 1, 998 (2011).

[7] V. Bhardwaj, E. Simova, P. Rajeev, C. Hnatovsky, R. Taylor, D. Rayner, and P. Corkum, "Optically produced arrays of planar nanostructures inside fused silica," Phys. Rev. Lett. 96, 057404 (2006).

[8] M. Beresna, M. Gecevičius, P. G. Kazansky, and T. Gertus, "Radially polarized optical vortex converter created by femtosecond laser nanostructuring of glass," Appl. Phys. Lett. 98, 201101 (2011).

[9] M.Beresna, M. Gecvicius, P.G. Kazansky, T. Taylor, and A. Kavokin, "Exciton mediated self-organization in glass driven by ultrashort light pulses," Appl. Phys. Lett. 101, 053120 (2012).

[10] Y. Shimotsuma, M. Sakakura, PG. Kazansky, M. Beresna, J. Qiu, K. Miura, and K. Hirao, "Ultrafast manipulation of self-assembled form birefringence in glass,” Advanced Materials 22 (36), 4039 (2010).

[11] http://www.wophotonics.com/products/accessories/radial-polarization-converter.

[12]P. G. Kazansky et al., "Quill" writing with ultrashort light pulses in transparent materials," Appl. Phys. Lett. 90, 151120 (2007).

[13]D. N. Vitek, E. Block, Y. Bellouard, D. E. Adams, S. Backus, D. Kleinfeld, C. G. Durfee, and J. A. Squier, "Spatiotemporally focused femtosecond laser pulses for nonreciprocal writing in optically transparent materials," Opt. Express 18, $24673(2010)$.

[14]P.G. Kazansky, Y. Shimotsuma, M. Sakakura, M. Beresna, M. Gecevicius, Yu. P. Svirko, S. Akturk, J. Qiu, K. Miura, and K. Hirao "Photosensitivity control of an isotropic medium through polarization of light pulses with tilted intensity front," Opt. Express 19, 20658 (2011). 